\title{
Comparing Long-Acting Antipsychotic Discontinuation Rates Under Ordinary Clinical Circumstances: A Survival Analysis from an Observational, Pragmatic Study
}

\author{
Federico Bertolini ${ }^{1}$ (1) - Giovanni Ostuzzi ${ }^{1} \cdot$ Michela Pievani $^{1} \cdot$ Andrea Aguglia $^{2,3}$. Francesco Bartoli ${ }^{4}$. \\ Paola Bortolaso $^{5}$. Camilla Callegari ${ }^{5}$. Mariarita Caroleo ${ }^{6}$. Giuseppe Carrà ${ }^{4,7}$. Mariangela Corbo ${ }^{8}$. \\ Armando D'Agostino ${ }^{9,10}$. Pasquale De Fazio ${ }^{11}$. Fabio Magliocco ${ }^{6}$. Giovanni Martinotti ${ }^{8}$.

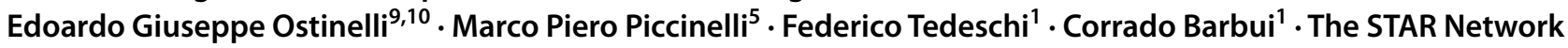 \\ Investigators
}

Accepted: 17 March 2021 / Published online: 29 March 2021

(c) The Author(s) 2021

\begin{abstract}
Background Recent guidelines suggested a wider use of long-acting injectable antipsychotics (LAI) than previously, but naturalistic data on the consequences of LAI use in terms of discontinuation rates and associated factors are still sparse, making it hard for clinicians to be informed on plausible treatment courses.

Objective Our objective was to assess, under real-world clinical circumstances, LAI discontinuation rates over a period of 12 months after a first prescription, reasons for discontinuation, and associated factors.

Methods The STAR Network 'Depot Study' was a naturalistic, multicentre, observational prospective study that enrolled subjects initiating a LAI without restrictions on diagnosis, clinical severity or setting. Participants from 32 Italian centres were assessed at baseline and at 6 and 12 months of follow-up. Psychopathology, drug attitude and treatment adherence were measured using the Brief Psychiatric Rating Scale, the Drug Attitude Inventory and the Kemp scale, respectively.

Results The study followed 394 participants for 12 months. The overall discontinuation rate at 12 months was 39.3\% (95\% confidence interval [CI] 34.4-44.3), with paliperidone LAI being the least discontinued LAI (33.9\%; 95\% CI 25.3-43.5) and olanzapine LAI the most discontinued (62.5\%; 95\% CI 35.4-84.8). The most frequent reason for discontinuation was onset of adverse events (32.9\%; 95\% CI 25.6-40.9) followed by participant refusal of the medication (20.6\%; 95\% CI 14.6-27.9). Medication adherence at baseline was negatively associated with discontinuation risk (hazard ratio [HR] $0.853 ; 95 \% \mathrm{CI}$ $0.742-0.981 ; p=0.026$ ), whereas being prescribed olanzapine LAI was associated with increased discontinuation risk compared with being prescribed paliperidone LAI (HR 2.156; 95\% CI 1.003-4.634; $p=0.049$ ).

Conclusions Clinicians should be aware that LAI discontinuation is a frequent occurrence. LAI choice should be carefully discussed with the patient, taking into account individual characteristics and possible obstacles related to the practicalities of each formulation.
\end{abstract}

\section{Introduction}

Antipsychotic discontinuation and lack of adherence represent major risk factors for relapse in people with both affective and non-affective psychoses and are key challenges for frontline practitioners [1]. Long-acting injectable (LAI)

Federico Bertolini, Giovanni Ostuzzi: Joint first authors.

Federico Bertolini

federico.bertolini@univr.it

Extended author information available on the last page of the article formulations of antipsychotics were developed in the early 1960s to enhance treatment adherence, although for many years they have been generally perceived as a last resort for patients with severe schizophrenia and even criticised as a coercive tool [2]. However, the evidence accompanying the introduction of second-generation antipsychotic (SGA) LAIs has led experts and guidelines to reconsider the role of these formulations, advocating for earlier and wider use, including in patients at their first episode of psychosis and in those with diagnoses other than schizophrenia [3-8]. In recent times, LAIs have been largely destigmatized by clinicians, and several strategies to address barriers towards LAI employment have been proposed [9]. 


\section{Key Points}

The STAR Network 'Depot Study' prospectively followed 394 subjects initiating treatment with long-acting injections (LAIs) of antipsychotics under naturalistic conditions for 12 months.

LAI discontinuation was frequent in everyday clinical practice in Italy, occurring in almost $40 \%$ of the entire sample; side effects, participant refusal to continue LAIs and LAIs no longer being required were the most frequently reported reasons for discontinuation.

Paliperidone LAI and aripiprazole LAI were the least discontinued medications (33.9 and 35.4\%, respectively), whereas more than half of participants initiating risperidone LAI and olanzapine LAI discontinued during the 12 months of follow-up (51.4 and 62.5\%, respectively).

In multivariate analysis, being prescribed olanzapine LAI and poor medication adherence at baseline were significantly associated with higher discontinuation risk.

However, whether different LAI medications are associated with different discontinuation rates and, more generally, with different patient outcomes, including attitudes towards medications, functioning and quality of life, remains uncertain [10]. Randomized controlled trials (RCTs) are usually regarded as the gold standard for evaluating the efficacy of medications, but in the case of LAIs they suffer from major limitations. A first issue is selection bias, as participants with low treatment adherence (who are likely to benefit the most from LAIs) might not be suitable for enrolment in RCTs [11]. Additionally, as study participation requires willingness to participate and adherence to complex procedures (e.g. double-dummy techniques), by definition, trial participants cannot be representative of difficult-to-treat patients who are generally reluctant to engage with mental health services [12]. Another concern is that RCTs require an artificial ecology that inevitably favours treatment adherence, and that is quite different from the real world of ordinary clinical practice [13].

Theoretically, observational studies, by following unselected cohorts of everyday patients over long periods of follow-up under everyday circumstances, may offer a better perspective to describe discontinuation rates associated with different LAI antipsychotics. Surprisingly, relatively few such studies have been conducted, and heterogeneous methodological approaches have been used, making it difficult to draw any clear conclusions. Some observational studies focused on one LAI antipsychotic only, comparing it with the corresponding oral medication or a mixture of oral antipsychotics, whereas studies comparing different LAIs with each other either did not include the most recently marketed SGA LAIs or did not explore sociodemographic and clinical characteristics possibly associated with LAI adherence [14-16].

Against this background, the present study was conducted to assess the proportion of subjects discontinuing LAI medications over a period of 12 months after an LAI prescription to ascertain whether different LAI medications are associated with different discontinuation rates, reasons for discontinuation and to explore factors possibly associated with the risk of discontinuation.

\section{Methods}

\subsection{Study Design and Inclusion Criteria}

This study was developed following the STROBE (STrengthening the Reporting of OBservational studies in Epidemiology) statement [17]. The full description of the methods of the STAR Network Depot Study and cross-sectional data analyses are reported elsewhere [18, 19]. Briefly, the STAR Network Depot Study was an observational, longitudinal multicentre study involving subjects initiating treatment with any LAI, consecutively recruited over a period of 12 months and assessed at 6 and 12 months of follow-up. Subjects were included if they were (1) adults (aged $\geq 18$ years), (2) willing to sign the informed consent, (3) initiating an LAI medication and (4) not receiving any other LAI during the previous 3 months. Given the pragmatic nature of the study, participants with any psychiatric diagnosis and any concomitant pharmacological treatment were included. Similarly, participants from any setting, including hospital psychiatric wards, community day centres and residential facilities, were included. Recruiting centres were part of the STAR Network (Servizi Territoriali Associati per la Ricerca-Community Services Associated for Research), an Italian research network aiming to gather original data from real-world clinical practice and provide new pragmatic insights for clinicians.

The study protocol was approved by the local ethics committees of recruiting centres and was made publicly available at the Open Science Framework online repository (https:// osf.io/wt8kx/). Participants provided written consent for the collection and analysis of their data. The STAR Network Depot Study was conducted independently of industry funding or support.

\subsection{Measures}

A standard form was used to collect sociodemographic (age, sex, education, marital status), clinical (diagnosis, number and days of hospitalizations during the last year, alcohol/ 
substance-related disorders, metabolic comorbidities) and pharmacological information (current and previous treatments, LAI prescribed, side effects). Clinical interviews, health records and chart reviews were used to collect participants' baseline data. A follow-up form was used to collect treatment data, including date and reasons (through checklist) for LAI discontinuation. In addition, the following rating scales were used: (1) The clinician-rated Brief Psychiatric Rating Scale (BPRS) [20], validated in the Italian language [21], assessed the overall level of psychiatric symptoms, with scores ranging from 31 to 40 indicating mild symptoms, from 41 to 52 moderate symptoms, and above 52 severe symptoms [22]; (2) the self-administered Drug Attitude Inventory 10 items (DAI-10) [23], validated in the Italian language [24], measured the subject's attitude toward medications, with scores ranging between -10 and 10 and higher scores indicating a better overall attitude toward medications; (3) the clinician-rated Kemp's 7-point scale [25], compiled by the clinician, assessed overall adherence to treatments, with scores ranging from 1 to 7 and higher scores indicating equally higher levels of adherence and scores of $\geq 5$ indicating an overall good acceptance of medications. These rating scales were administered at baseline and after 6 and 12 months of follow-up.

\subsection{Data Management and Statistical Analysis}

Baseline and follow-up data were periodically forwarded from each recruiting centre to the coordinating centre (University of Verona), where they were entered into a computer database. Data correctness and consistency were ensured with the use of a double-entry technique and by applying a set of electronic and manual edit checks. Participants' data were recorded anonymously. A unique number for each participant was used both in the recruitment and the follow-up forms, and confidentiality was guaranteed throughout the entire course of the study, in accordance with the Declaration of Helsinki [26].

Continuous variables were expressed as means and standard deviations, whereas categorical variables were expressed as absolute numbers and percentages. Statistical significance was assessed using the Mann-Whitney $U$ test for continuous variables, and through the chi-squared test or Fisher's exact test as appropriate for categorical variables. A Kaplan-Meier survival curve was plotted to describe the proportion of participants discontinuing first-generation antipsychotics (FGAs), considered as a group, and each SGA LAI over the time. To identify factors associated with LAI discontinuation, a multiple regression, including a random effect per centre, was performed. As the variance of this random effect was not significantly different from 0 , we then performed non-parametric Cox regressions without a random effect. Statistical analyses were performed with the software Stata version 15.1 [27].

\section{Results}

\subsection{Participant Characteristics}

The study included 461 subjects initiating treatment with an LAI, recruited in 35 Italian centres. As three recruiting centres contributed only to the cross-sectional phase of the study, 31 participants were not included in the longitudinal assessment, and 36 additional participants refused to give consent for the follow-up assessment. Overall, 394 participants recruited in 32 centres provided data for the survival analysis. Figure 1 presents the number of participants and their distribution among the diagnostic categories for each LAI. The majority of the sample received SGA LAIs (67\%). Paliperidone LAI (once monthly) was the most frequently prescribed medication, followed by aripiprazole LAI (aripiprazole monohydrate), haloperidol LAI, risperidone LAI, fluphenazine LAI, olanzapine LAI, zuclopenthixol LAI and perphenazine LAI. Schizophrenia was the most common diagnosis, accounting for more than $50 \%$ of study participants.

During the 1-year follow-up, a total of 155 participants discontinued treatment with the LAI prescribed at baseline, yielding a discontinuation rate of $39.3 \%$ (95\% confidence interval [CI] 34.5-44.4). Table 1 presents the main baseline sociodemographic and clinical characteristics of participants continuing and discontinuing treatment with LAI antipsychotics. Compared with those who did not discontinue, participants discontinuing treatment during follow-up had poorer attitudes towards medications (as measured by the DAI-10 scale) and were considered less adherent (as measured by the Kemp scale). Discontinuers were significantly older (by an average of approximately 2 years), although illness duration was slightly shorter than in continuers. Diagnostic categories were balanced between continuers and discontinuers, and no significant differences were found in terms of BPRS scores, although more participants discontinuing treatment had been involuntarily hospitalized prior to the study ${ }^{1}$.

\subsection{Survival Probability for Long-Acting Injectable (LAI) Antipsychotics}

The Kaplan-Meier survival estimate showed that paliperidone LAI was the least discontinued antipsychotic (33.9\%; 95\% CI 25.3-43.5), followed by aripiprazole LAI (35.4\%;

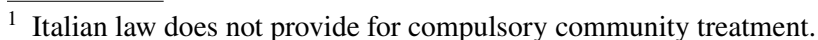




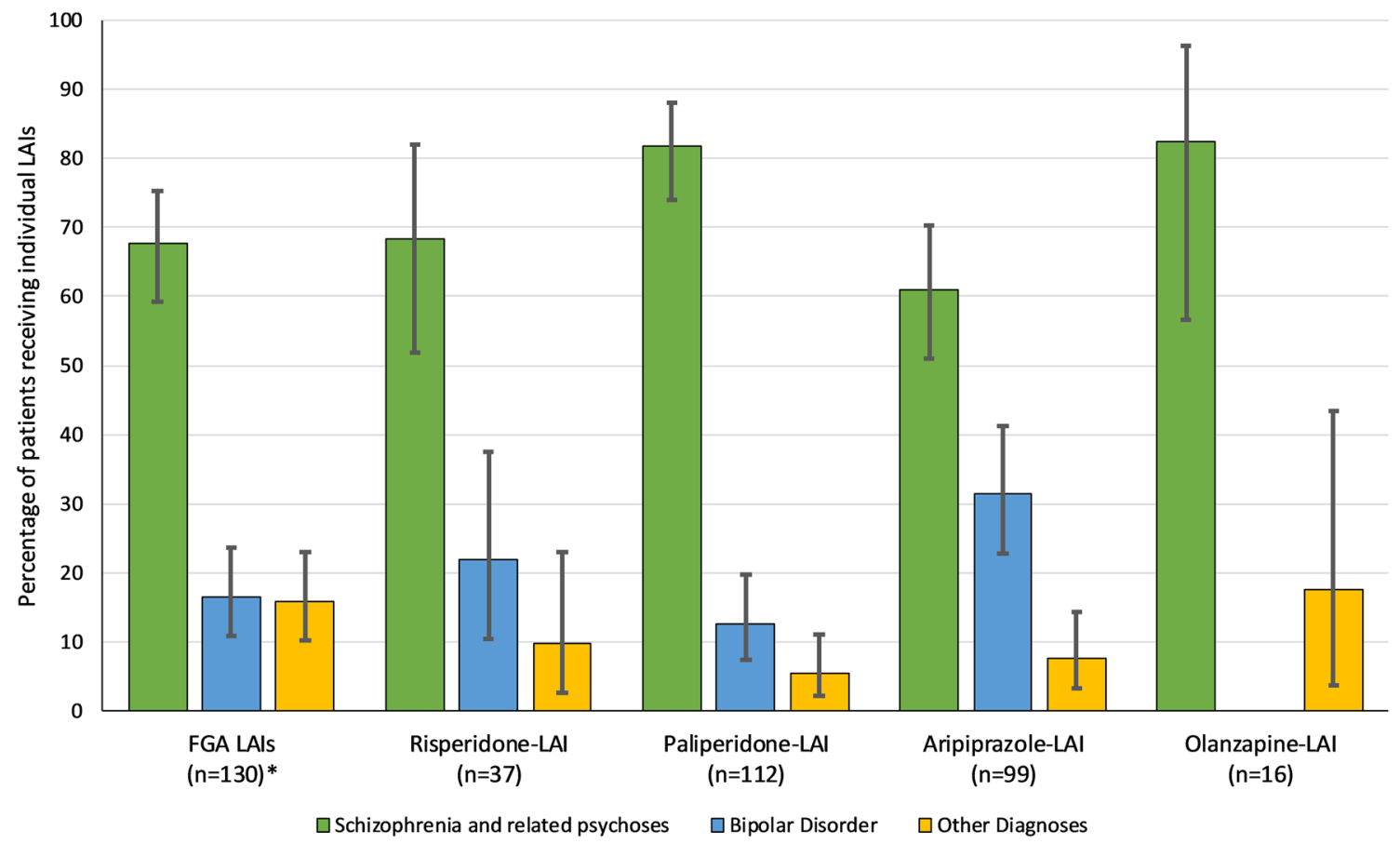

Fig. 1 Distribution of participants receiving a first long-acting injectable antipsychotic prescription by diagnostic category. Error bars represent $95 \%$ confidence intervals. FGA first-generation antipsychotic,

95\% CI 26.0-45.3) and by the group of FGA LAIs (40.8\%; 95\% CI 32.2-49.7). Risperidone LAI (51.4\%; 95\% CI 34.4-68.1) and olanzapine LAI (62.5\%; 95\% CI 35.4-84.8) were the only antipsychotics with a discontinuation rate greater than $50 \%$ and had a significantly higher hazard of discontinuation than paliperidone LAI, the best-performing agent (Fig. 2). Table S1 in the electronic supplementary material (ESM) shows the discontinuation rates for each LAI.

\subsection{Reasons for LAI Discontinuation}

The most frequently reported reason for LAI discontinuation was the occurrence of adverse events $(32.9 \%$; $95 \%$ CI 25.6-40.9), followed by participant refusal of continuing treatment $(20.6 \%$; 95\% CI 14.6-27.9) and the clinician considering the LAI to be no longer required $(20.0 \%$; $95 \%$ CI 14.0-27.2). LAI inefficacy was the least frequently reported reason for discontinuation (17.4\%; 95\% CI 11.8-24.3) (Table 2). Adverse events that led to LAI discontinuation, and discontinuation reasons for each LAI, are reported in Tables S2 and S3 in the ESM, respectively.

\subsection{Factors Associated with LAI Discontinuation}

After adjustment for a number of confounders, non-parametric Cox regression analysis revealed that receiving
LAI long-acting injectable antipsychotic. *Haloperidol LAI $n=85$, fluphenazine LAI $n=27$, zuclopenthixol LAI $n=16$, perphenazine LAI $n=2$

olanzapine LAI, as compared with paliperidone LAI, the best-performing agent, significantly increased the risk of discontinuation. Lower adherence at baseline, as measured by the Kemp scale, was additionally associated with a higher risk of discontinuation (Table 3 ).

\section{Discussion}

This study described the longitudinal time course of LAI discontinuation in an unselected group of subjects enrolled in the context of everyday clinical practice by an Italian network of mental health services. Study findings showed that LAIs are commonly prescribed to people with a broad spectrum of diagnoses, including not only schizophrenia but also bipolar disorder, personality disorders and organic mental disorders, although a registered therapeutic indication by the Italian Medicines Agency exists only for schizophrenia. Most of the participants received SGA LAIs, with paliperidone and aripiprazole being the most frequent choices, followed by the FGA LAI haloperidol. Notably, participants were recruited in a variety of settings and types of mental health services, including outpatient, inpatient, academic and non-academic facilities. This choice was made to resemble real-world practice insofar as possible. 
Table 1 Baseline sociodemographic and clinical characteristics of participants continuing and discontinuing long-acting antipsychotics over the 12 months after first prescription

\begin{tabular}{|c|c|c|c|}
\hline \multirow[t]{2}{*}{ Characteristic } & \multicolumn{2}{|l|}{ Discontinuers } & \multirow[t]{2}{*}{$p$-Value } \\
\hline & No $(n=239)$ & Yes $(n=155)$ & \\
\hline Age & $41.6 \pm 13.1$ & $43.8 \pm 13.2$ & 0.001 \\
\hline Women & $86(36.0)$ & $67(43.2)$ & 0.150 \\
\hline Italian nationality & $214(90.3)$ & $135(87.1)$ & 0.322 \\
\hline At least moderate housing self-sufficiency & 89 (37.2) & $78(50.3)$ & $\mathbf{0 . 0 1 0}$ \\
\hline Married & $35(14.6)$ & $20(12.9)$ & 0.626 \\
\hline High school or higher education & $113(47.9)$ & $78(51.0)$ & 0.550 \\
\hline Employed & $51(21.3)$ & $33(21.3)$ & 0.991 \\
\hline \multicolumn{4}{|l|}{ Diagnosis } \\
\hline Schizophrenia & $126(52.7)$ & $82(52.9)$ & 0.152 \\
\hline Schizoaffective disorder & $38(15.9)$ & $32(20.6)$ & \\
\hline Bipolar disorder & $52(21.8)$ & $22(14.2)$ & \\
\hline Personality disorders & $11(4.6)$ & $14(9.0)$ & \\
\hline Organic mental disorders ${ }^{\mathrm{a}}$ & $8(3.3)$ & $3(1.9)$ & \\
\hline Obsessive-compulsive disorder & $2(0.8)$ & $2(1.3)$ & \\
\hline Years of illness & $12.6 \pm 9.8$ & $10.9 \pm 10.3$ & 0.014 \\
\hline Alcohol use & $39(16.3)$ & $23(14.8)$ & 0.694 \\
\hline Any substance use & $50(20.9)$ & $32(20.6)$ & 0.948 \\
\hline At least one medical comorbidity & $75(31.4)$ & $44(28.4)$ & 0.527 \\
\hline At least one hospitalization in the last year & $138(57.7)$ & $102(65.8)$ & 0.109 \\
\hline At least one compulsory hospitalization & $40(16.8)$ & $39(25.2)$ & 0.043 \\
\hline Psychotropic drug dose, PDD/DDD & $1.2 \pm 2.0$ & $0.9 \pm 0.8$ & 0.319 \\
\hline LAIs in the past & $75(31.4)$ & $45(29.0)$ & 0.621 \\
\hline BPRS & $48.7 \pm 14.0$ & $49.7 \pm 14.4$ & 0.436 \\
\hline DAI-10 & $2.5 \pm 5.2$ & $1.2 \pm 5.3$ & $\mathbf{0 . 0 2 1}$ \\
\hline Kemp's 7-point scale & $5.0 \pm 1.5$ & $4.4 \pm 1.4$ & $<0.001$ \\
\hline
\end{tabular}

Data are presented as mean \pm standard deviation or $n(\%)$ unless otherwise indicated. Bold formatting represents statistically significant results $B P R S$ Brief Psychiatric Rating Scale, DAI Drug Attitude Inventory, DDD defined daily dose, LAIs long-acting injectables, $n$ number of participants, $P D D$ prescribed daily dose

${ }^{a}$ Includes dementia, mental organic disorder, mental retardation and drug-induced psychosis

Despite being one of the most relevant strengths of this study, it also complicates the comparison with available literature, as differences in terms of study methods, local clinical practice and socio-economic context may influence point estimates of discontinuation rates and associated factors.

The finding of an overall discontinuation rate of around $40 \%$ over 12 months of follow-up, with olanzapine LAI and risperidone LAI showing the highest discontinuation rates ( 62.5 and $51.4 \%$, respectively), takes place among the variable retention rates previously reported. Using administrative prescription databases in the Netherlands and Belgium, Decuypere et al. [28] documented discontinuation rates above $50 \%$ after 12 months of follow-up for paliperidone once monthly, risperidone LAI, olanzapine LAI and haloperidol LAI. Higher treatment continuation of paliperidone once monthly compared with risperidone LAI and haloperidol decanoate were observed in both countries, together with a significantly higher treatment continuation of paliperidone once monthly compared with olanzapine LAI in the Netherlands. Among British cohorts of patients beginning LAI treatment, discontinuation rates at 1 year were $35-40 \%$ for paliperidone once monthly [29-31] and $68-55 \%$ for risperidone LAI [32, 33]. Iwata et al. [15] found discontinuation rates for aripiprazole of around $40 \%$ among patients with schizophrenia in Japan at 12 months of follow-up [15], whereas, in Canada, treatment retention at 18 months was $77 \%$ for a group of 70 patients with schizophrenia initiated with risperidone LAI [34]. On the other hand, in the USA, Pilon et al. [35] found notably higher discontinuation rates, with more than $60 \%$ of patients with schizophrenia using aripiprazole LAI, paliperidone once monthly and risperidone LAI discontinuing treatment after 12 months. Another study conducted in the USA reported that around $75 \%$ of patients with schizophrenia and bipolar disorder who initiated 
Fig. 2 Kaplan-Meier survival estimate of the probability of continuing antipsychotic longacting injectable antipsychotics during the 12 months after initial prescription. *Statistically significant difference between risperidone LAI and paliperidone LAI (HR 2.014; 95\% CI 1.152-3.522; $p=0.014)$. $* *$ Statistically significant difference between olanzapine LAI and paliperidone LAI (HR 2.363; 95\% CI 1.170-4.774; $p=0.017) . C I$ confidence interval, $F G A$ first-generation antipsychotic, $H R$ hazard ratio, LAI long-acting injectable antipsychotic

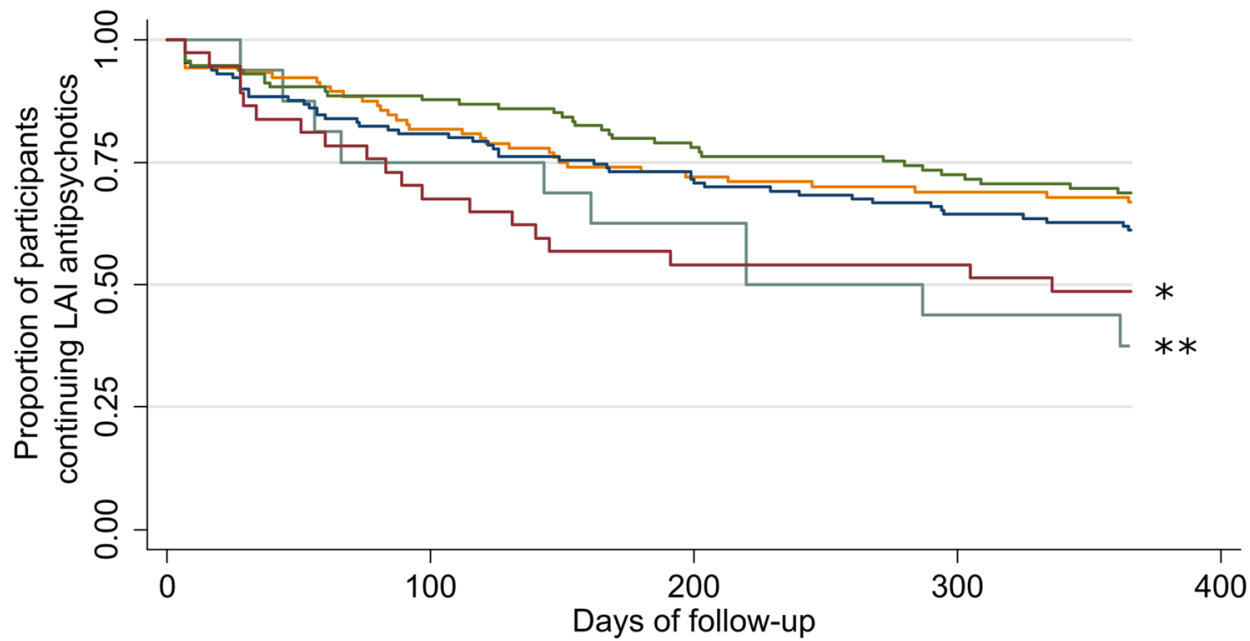

FGA LAls

Paliperidone-LAI

Olanzapine-LAI
Risperidone-LAI

Aripiprazole-LAI
Table 2 Reasons for discontinuing long-acting antipsychotics over the 12 months after first prescription

\begin{tabular}{lll}
\hline Reason & $n$ & $\%(95 \%$ CI $)$ \\
\hline Adverse events & 51 & $32.9(25.6-40.9)$ \\
Participant's refusal & 32 & $20.6(14.6-27.9)$ \\
LAIs no longer required & 31 & $20.0(14.0-27.2)$ \\
Inefficacy & 27 & $17.4(11.8-24.3)$ \\
\hline
\end{tabular}

This information was not available for 14 participants

$C I$ confidence interval, LAI long-acting injectable antipsychotic, $n$ number of participants

aripiprazole LAI discontinued treatment after 1 year [14]. In Austria, Rittmannsberger et al. [36], who analysed a total of 194 treatment episodes with new start of LAIs, found that almost one-half of patients dropped out after 6 months of follow-up.

The finding of higher discontinuation rates for olanzapine LAI and risperidone LAI compared with the best-performing agent, paliperidone LAI, reinforces the notion that very pragmatic reasons may influence treatment adherence in clinical practice. For olanzapine LAI, the risk of postinjection delirium syndrome and the mandatory 3 -h postadministration monitoring period in appropriate facilities are important barriers to long-term use, and similar considerations might apply to LAI medications requiring bimonthly administration, such as risperidone LAI.

Occurrence of adverse events was reported as the most frequent reason for LAI discontinuation, whereas lack of efficacy was seldom reported. These results are not in line with those from several similar pharmacoepidemiological studies, in which lack of effectiveness played a substantial role as reason for discontinuation. For instance, in a catchment area of south London, $52 \%$ of a sample of patients on paliperidone palmitate discontinued treatment for lack of effectiveness, whereas adverse events accounted for $14.5 \%$ of discontinuations [31]. In the same catchment area, for risperidone LAI, Taylor et al. [37] reported lack of efficacy and adverse events in 38 and $29 \%$ of those who discontinued, respectively. A possible explanation for the high proportion of discontinuations related to adverse events observed in the present study may be that many participants initiated LAI treatment without previous treatment with the oral formulation of the same antipsychotic. This might have increased the proportion of discontinuers due to adverse effects. However, the Cox regression did not support this explanation, as it did not find any association between absence of previous treatment with oral formulation and discontinuation risk. This suggests that further factors may have influenced tolerability over time (e.g. dose adjustments) and that clinicians should be continuously alerted to the occurrence of clinically relevant side effects, even after several months of treatment.

Interestingly, around $20 \%$ of LAI discontinuations was motivated by the LAI formulation being no longer required, which may reflect that individual needs and preferences may vary over time, for instance due to progressive improvement of the underlying psychopathology, increased illness awareness and/or improved attitude towards medications, but also environmental changes, such as psycho-social support or admission to residential programmes with supervised drug administration. Therefore, in these cases, discontinuation should not be considered a negative outcome, highlighting the relevance of periodically reconsidering the 
Table 3 Factors associated with the hazard of discontinuing long-acting antipsychotics over the 12 months after first prescription: multiple Cox regression analysis

\begin{tabular}{|c|c|c|c|}
\hline \multirow[t]{2}{*}{ Variables } & \multicolumn{3}{|c|}{ Multiple regression $(n=390)$} \\
\hline & $\mathrm{HR}$ & $95 \% \mathrm{CI}$ & $p$-Value \\
\hline Age (years) & 1.009 & $0.991-1.026$ & 0.326 \\
\hline Female sex & 0.965 & $0.652-1.429$ & 0.858 \\
\hline Not Italian & 1.024 & $0.576-1.823$ & 0.937 \\
\hline At least moderate housing self-sufficiency & 1.444 & $0.948-2.200$ & 0.087 \\
\hline High school or higher education & 1.113 & $0.785-1.578$ & 0.549 \\
\hline Employed & 1.136 & $0.745-1.732$ & 0.554 \\
\hline $\mathrm{PDD} / \mathrm{DDD}$ & 0.946 & $0.850-1.052$ & 0.305 \\
\hline Alcohol use & 0.706 & $0.406-1.225$ & 0.215 \\
\hline Substance use & 1.305 & $0.791-2.152$ & 0.298 \\
\hline Number of medical comorbidities & 0.871 & $0.571-1.327$ & 0.520 \\
\hline BPRS score & 0.998 & $0.984-1.011$ & 0.766 \\
\hline DAI-10 score & 0.983 & 0.949-1.019 & 0.358 \\
\hline Kemp score & 0.849 & $0.739-0.976$ & 0.021 \\
\hline Number of LAIs in the past & 0.846 & $0.657-1.089$ & 0.194 \\
\hline Cumulative days of hospitalizations in the last year & 0.998 & $0.988-1.008$ & 0.691 \\
\hline Compulsory admissions in the last year & 0.888 & $0.567-1.391$ & 0.603 \\
\hline Number of previous antipsychotic drugs & 0.809 & $0.384-1.705$ & 0.577 \\
\hline Prior clozapine use & 1.680 & $0.825-3.419$ & 0.152 \\
\hline Inpatient when starting LAI & 1.050 & $0.726-1.520$ & 0.794 \\
\hline Oral trial before LAI administration & 0.803 & $0.519-1.244$ & 0.327 \\
\hline \multicolumn{4}{|l|}{ LAI } \\
\hline Paliperidone LAI & 1 (ref. & & \\
\hline FGA LAIs & 0.981 & $0.606-1.588$ & 0.937 \\
\hline Risperidone LAI & 1.581 & $0.855-2.921$ & 0.144 \\
\hline Aripiprazole LAI & 1.220 & $0.731-2.037$ & 0.446 \\
\hline Olanzapine LAI & 2.367 & $1.058-5.295$ & 0.036 \\
\hline \multicolumn{4}{|l|}{ Diagnosis } \\
\hline Schizophrenia and related psychosis & 1 (ref. & & \\
\hline Bipolar disorder & 0.674 & $0.409-1.111$ & 0.122 \\
\hline Other diagnoses & 1.295 & $0.751-2.234$ & 0.352 \\
\hline Prescriber's age & 0.998 & $0.982-1.015$ & 0.813 \\
\hline
\end{tabular}

Bold formatting represents statistically significant results

$B P R S$ Brief Psychiatric Rating Scale, $C I$ confidence interval, DAI Drug Attitude Inventory, $D D D$ defined daily dose, $F G A$ first-generation antipsychotic, $H R$ hazard ratio, $L A I s$ long-acting injectables, $n$ number of participants, $P D D$ prescribed daily dose, $r e f$. reference

appropriateness of current therapies, without considering LAIs only as a last resort approach with no return. Interestingly, other studies reported lower rates of discontinuation motivated by the LAI formulation being no longer required. For instance, Deslandes et al. [29] reported that only 13\% of a sample of patients receiving risperidone LAI discontinued treatment based on patient choice or request; similarly, Attard et al. [31] found that only $17 \%$ of a sample of patients receiving paliperidone palmitate discontinued treatment based on patient preferences and choices. In the context of the present study, it is possible that cultural factors related to generic negative views that LAI formulations often have among doctors and patients motivated higher proportions of discontinuations based on reasons not related to efficacy or tolerability, but, rather, related to patients' views and requests.

Although participants' refusal to continue the LAI medication accounted for only about one-fifth of discontinuations, the present study showed that the level of adherence at baseline was associated with discontinuation risk over the subsequent 12 months of follow-up. Of note, no other sociodemographic or clinical characteristics emerged as relevant factors. In particular, no diagnosis was associated with a higher likelihood of LAI discontinuation. Within this cohort, 
we could not confirm some previously reported predictors of discontinuation, such as inpatient status at LAI initiation $[31,38]$, having had an adequate oral trial [30] or history of treatment resistance (in the form of number of previous antipsychotics trials or prior clozapine use) [39]. The studies that found these variables as predictors did consider cohorts initiated on paliperidone once monthly or risperidone LAI and considered specific local areas. It is possible that not all LAIs share these predictors or that our multicentre cohort was underpowered to detect these associations. However, other single-centre studies (where overall heterogeneity can be expected to be considerably lower than in our study) were also unable to find discontinuation predictors [29, 30].

Limitations of the present study are those related to the naturalistic approach employed. We acknowledge that, although discontinuation rate may be considered a highly pragmatic outcome measure, especially in difficult-to-treat patient populations such as those with psychotic disorders, lack of an experimental design prevents us from drawing conclusions related to the comparative efficacy of LAIs. However, the present study can inform on the practical consequences of initiating different LAI medications in realworld settings. A second limitation is that, for some LAIs, particularly olanzapine LAI and risperidone LAI, the number of participants was relatively low, limiting the precision of our estimates. Moreover, a larger sample size would have enabled analysis of the reasons leading to individual LAI discontinuations. The regulatory requirement for written informed consent for data collection might have led to noninclusion of some patients (e.g., those affected by severe paranoid symptoms). However, the baseline clinical characteristics do not suggest a lack of representation of subjects with severe symptoms. An additional shortcoming is that, at the time the study was conducted, paliperidone 3-monthly LAI was not available in Italy.

\section{Conclusions}

Clinicians should be aware that LAI discontinuation is a frequent occurrence in clinical practice and that regular assessment of adherence levels before and during LAI treatment might be helpful to predict and monitor this risk. The choice of the LAI should be carefully discussed with the patient, taking into account not only the efficacy and tolerability profile in light of the patient's individual characteristics but also possible obstacles related to the practicality of the formulation. In particular, clinicians should explicitly ask about patient and caregiver views on the interval between administrations, the need for oral supplementation, the need for clinical observation after injection, any fear of needles and the expected tolerance to local site pain. Our results suggest that individuals initiated on olanzapine LAI and risperidone
LAI may experience higher discontinuation rates than those receiving other LAI medications.

Supplementary Information The online version contains supplementary material available at https://doi.org/10.1007/s40263-021-00809-w.

Acknowledgements The STAR Network Investigators are as follows: Corrado Barbui, Federico Bertolini, Filippo Boschello, Chiara Gastaldon, Maria Angela Mazzi, Michela Nosé, Giovanni Ostuzzi, Davide Papola, Giovanni Perini, Alberto Piccoli, Michela Pievani, Marianna Purgato, Mirella Ruggeri, Federico Tedeschi, Samira Terlizzi and Giulia Turrini (Verona); Mariarita Caroleo, Pasquale De Fazio, Fabio Magliocco and Gaetano Raffaele (Catanzaro); Simone Cavallotti, Margherita Chirico, Armando D’Agostino, Farida Ferrato, Ivan Limosani, Daniele Mastromo, Emiliano Monzani, Edoardo Giuseppe Ostinelli, Matteo Porcellana and Francesco Restaino (Milano); Pasqua Maria Annese, Simone Bolognesi, Massimiliano Cerretini, Alberto De Capua, Sara Debolini, Maria Del Zanna, Francesco Fargnoli, Alessandra Giannini, Livia Luccarelli, Claudio Lucii, Elisa Pierantozzi and Fiorella Tozzi (Siena); Francesco Bardicchia, Giuseppe Cardamone, Edvige Facchi, Nadia Magnani and Federica Soscia (Grosseto); Bruno Biancosino and Spyridon Zotos (Ferrara); Marzio Giacomin, Francesco Pompei, Mariangela Spano and Filippo Zonta (Treviso); Aldo Buzzi, Camilla Callegari, Roberta Calzolari, Ivano Caselli, Marcello Diurni, Edoardo Giana, Marta Ielmini, Anna Milano, Nicola Poloni, Emanuele Sani and Daniele Zizolfi (Varese); Gabrio Alberini, Paola Bortolaso, Sara Cazzamalli, Chiara Costantini, Angela Di Caro, Chiara Paronelli, Silvia Piantanida and Marco Piccinelli (Varese Verbano); Papalini Alessandro, Silva Veronica Barbanti, Chiara D’Ippolito, Mauro Gozzi and Valentina Moretti (Reggio Emilia); Ornella Campese, Mariangela Corbo, Lucia Di Capro, Massimo di Giannantonio, Federica Fiori, Marco Lorusso, Valerio Mancini, Giovanni Martinotti and Daniela Viceconte (Chieti); Carmela Calandra, Maria Luca, Maria Salvina Signorelli and Francesco Suraniti (Catania); Beatrice Balzarro, Giancarlo Boncompagni, Valentina Caretto, Roberta Emiliani, Pasqualino Lupoli, Marco Menchetti, Eugenio Rossi, Viviana Storbini, Ilaria Tarricone and Laura Terzi (Bologna); Marianna Boso, Cristina Catania, Giuseppe De Paoli and Paolo Risaro (Pavia); Flora Aspesi, Francesco Bartoli, Mattia Bava, Adele Bono, Giulia Brambilla, Giuseppe Carrà, Gloria Castagna, Sara Lucchi, Roberto Nava, Milena Provenzi, Tommaso Tabacchi, Martina Tremolada and Enrica Verrengia (Monza); Michela Barchiesi and Maria Ginevra Oriani (Ancona); Monica Pacetti (Forlì); Andrea Aguglia, Maurizio Ferro and Lucio Ghio (Genova); Rossella Beneduce, Laura Laffranchini, Laura Rosa Magni, Giuseppe Rossi and Giovanni Battista Tura (Brescia); Lelio Addeo, Giovanni Balletta, Elisa De Vivo, Rossella Di Benedetto and Vincenzo Fricchione Parise (Avellino); Bernardo Carpiniello and Federica Pinna (Cagliari); Damiano Pecile (Mantova); Chiara Mattei (Fermo); Tommaso Bonavigo, Elisabetta Pascolo Fabrici, Sofia Panarello, Giulia Peresson and Claudio Vitucci (Trieste); Monica Pacetti (Forlì); Francesco Gardellin and Stefania Strizzolo (Vicenza); Edoardo Cossetta, Carlo Fizzotti and Daniele Moretti (Savona); Luana Di Gregorio and Francesca Sozzi (Trento); Giuseppe Colli and Daniele La Barbera (Palermo); and Sabrina Laurenzi (Civitanova Marche).

\section{Declarations}

Funding Open access funding provided by Università degli Studi di Verona within the CRUI-CARE Agreement.

Conflicts of Interest Giovanni Martinotti has been a consultant and/ or a speaker and/or has received research grants from Angelini, Doc Generici, Janssen, Lundbeck, Otsuka, and Pfizer. Federico Bertolini, 
Giovanni Ostuzzi, Michela Pievani, Andrea Aguglia, Francesco Bartoli, Paola Bortolaso, Camilla Callegari, Mariarita Caroleo, Giuseppe Carrà, Mariangela Corbo, Armando D'Agostino, Pasquale De Fazio, Fabio Magliocco, Edoardo Giuseppe Ostinelli, Marco Piero Piccinelli, Federico Tedeschi and Corrado Barbui have no conflicts of interest that are directly relevant to the content of this article.

Availability of data and material The study dataset is available from the authors on reasonable request.

Ethics approval The study protocol was approved by the local ethics committee of the coordinating centre (Ethics Committee for Clinical Trials of the Provinces of Verona and Rovigo, protocol n. 57622 of the 09/12/2015) and of each participating centre.

Consent to participate Participants provided written consent to data collection.

Code availability Not applicable.

Author contributions $\mathrm{GO}, \mathrm{GC}, \mathrm{GM}$ and $\mathrm{CB}$ contributed to study design. GO, FT and CB conducted the statistical analysis. All authors contributed to interpretation of the data. FB, GO and $\mathrm{CB}$ wrote the manuscript. All authors reviewed and commented on manuscript drafts and approved the final article and the decision to submit it for publication.

Open Access This article is licensed under a Creative Commons Attribution-NonCommercial 4.0 International License, which permits any non-commercial use, sharing, adaptation, distribution and reproduction in any medium or format, as long as you give appropriate credit to the original author(s) and the source, provide a link to the Creative Commons licence, and indicate if changes were made. The images or other third party material in this article are included in the article's Creative Commons licence, unless indicated otherwise in a credit line to the material. If material is not included in the article's Creative Commons licence and your intended use is not permitted by statutory regulation or exceeds the permitted use, you will need to obtain permission directly from the copyright holder. To view a copy of this licence, visit http://creativecommons.org/licenses/by-nc/4.0/.

\section{References}

1. Bowtell M, Ratheesh A, McGorry P, Killackey E, O'Donoghue B. Clinical and demographic predictors of continuing remission or relapse following discontinuation of antipsychotic medication after a first episode of psychosis. A systematic review. Schizophr Res. 2018;197:9-18. https://doi.org/10.1016/j.schres.2017.11.010.

2. Taylor DM, Velaga S, Werneke U. Reducing the stigma of long acting injectable antipsychotics - current concepts and future developments. Nord J Psychiatry. 2018;72(sup1):S36-9. https:// doi.org/10.1080/08039488.2018.1525638.

3. Barnes TR, Drake R, Paton C, Cooper SJ, Deakin B, Ferrier IN, et al. Evidence-based guidelines for the pharmacological treatment of schizophrenia: updated recommendations from the British Association for Psychopharmacology. J Psychopharmacol. 2020;34(1):3-78. https://doi.org/10.1177/0269881119889296.

4. Galletly C, Castle D, Dark F, Humberstone V, Jablensky A, Killackey E, et al. Royal Australian and New Zealand College of Psychiatrists clinical practice guidelines for the management of schizophrenia and related disorders. Aust N Z J Psychiatry. 2016;50(5):410-72. https://doi.org/10.1177/0004867416641195.
5. NICE. Psychosis and schizophrenia in adults: prevention and management. Clinical guideline [CG178]. 2014. https://www.nice.org. uk/guidance/cg178. Accessed 1 Sept 2020.

6. SIGN. Management of schizophrenia. A national clinical guideline. Edinburgh: Scottish Intercollegiate Guidelines Network; 2013.

7. Stahl SM. Long-acting injectable antipsychotics: shall the last be first? CNS Spectr. 2014;19(1):3-5. https://doi.org/10.1017/s1092 852913001016.

8. Stevens GL, Dawson G, Zummo J. Clinical benefits and impact of early use of long-acting injectable antipsychotics for schizophrenia. Early Interv Psychiatry. 2016;10(5):365-77. https://doi.org/ 10.1111/eip.12278.

9. Lindenmayer JP, Glick ID, Talreja H, Underriner M. Persistent barriers to the use of long-acting injectable antipsychotics for the treatment of Schizophrenia. J Clin Psychopharmacol. 2020;40(4):346-9. https://doi.org/10.1097/jcp.0000000000 001225 .

10. Zhao YJ, Lin L, Teng M, Khoo AL, Soh LB, Furukawa TA, et al. Long-term antipsychotic treatment in schizophrenia: systematic review and network meta-analysis of randomised controlled trials. BJPsych Open. 2016;2(1):59-66. https://doi.org/10.1192/bjpo.bp. 115.002576.

11. Kishimoto T, Hagi K, Nitta M, Leucht S, Olfson M, Kane JM, et al. Effectiveness of long-acting injectable vs oral antipsychotics in patients with schizophrenia: a meta-analysis of prospective and retrospective cohort studies. Schizophr Bull. 2018;44(3):603-19. https://doi.org/10.1093/schbul/sbx090.

12. Hofer A, Hummer M, Huber R, Kurz M, Walch T, Fleischhacker WW. Selection bias in clinical trials with antipsychotics. J Clin Psychopharmacol. 2000;20(6):699-702. https://doi.org/10.1097/ 00004714-200012000-00019.

13. Davis JM, Metalon L, Watanabe MD, Blake L, Metalon L. Depot antipsychotic drugs. Place in therapy. Drugs. 1994;47(5):741-73. https://doi.org/10.2165/00003495-199447050-00004.

14. Yan T, Greene M, Chang E, Hartry A, Touya M, Broder MS. Medication adherence and discontinuation of aripiprazole oncemonthly $400 \mathrm{mg}$ (AOM 400) versus oral antipsychotics in patients with schizophrenia or bipolar I disorder: a real-world study using US claims data. Adv Ther. 2018;35(10):1612-25. https://doi.org/ 10.1007/s12325-018-0785-y.

15. Iwata N, Inagaki A, Sano H, Niidome K, Kojima Y, Yamada S. Treatment persistence between long-acting injectable versus orally administered aripiprazole among patients with schizophrenia in a real-world clinical setting in Japan. Adv Ther. 2020;37(7):332436. https://doi.org/10.1007/s12325-020-01396-w.

16. Tiihonen J, Mittendorfer-Rutz E, Majak M, Mehtälä J, Hoti F, Jedenius E, et al. Real-world effectiveness of antipsychotic treatments in a nationwide cohort of 29823 patients with schizophrenia. JAMA Psychiat. 2017;74(7):686-93. https://doi.org/10.1001/ jamapsychiatry.2017.1322.

17. von Elm E, Altman DG, Egger M, Pocock SJ, Gøtzsche PC, Vandenbroucke JP. The Strengthening the Reporting of Observational Studies in Epidemiology (STROBE) statement: guidelines for reporting observational studies. J Clin Epidemiol. 2008;61(4):344-9. https://doi.org/10.1016/j.jclinepi.2007.11.008.

18. Ostuzzi G, Mazzi MA, Terlizzi S, Bertolini F, Aguglia A, Bartoli $\mathrm{F}$, et al. Factors associated with first-versus second-generation long-acting antipsychotics prescribed under ordinary clinical practice in Italy. PLoS ONE. 2018;13(8):e0201371. https://doi.org/10. 1371/journal.pone.0201371.

19. Bartoli F, Ostuzzi G, Crocamo C, Corbo M, D’Agostino A, Martinotti $\mathrm{G}$, et al. Clinical correlates of paliperidone palmitate and aripiprazole monohydrate prescription for subjects with schizophrenia-spectrum disorders: findings from the STAR Network 
Depot Study. Int Clin Psychopharmacol. 2020;35(4):214-20. https://doi.org/10.1097/yic.0000000000000317.

20. Overall J. The brief psychiatric rating scale in psychopharmacology research. In: Olivier-Martin R, editor. Pichot P. S. Karger AG; 1974. p. 67-78.

21. Roncone R, Tozzini C, Mazza M, de Risio A, Giosuè P, Morosini $\mathrm{P}$, et al. Validation of the Italian version of the Self-report Insight Scale. Epidemiol Psichiatr Soc. 2003;12(1):63-75. https://doi.org/ 10.1017/s1121189x00006060.

22. Leucht S, Kane JM, Kissling W, Hamann J, Etschel E, Engel R. Clinical implications of Brief Psychiatric Rating Scale scores. Br J Psychiatry. 2005;187:366-71. https://doi.org/10.1192/bjp.187.4. 366.

23. Hogan TP, Awad AG, Eastwood R. A self-report scale predictive of drug compliance in schizophrenics: reliability and discriminative validity. Psychol Med. 1983;13(1):177-83. https://doi.org/10. 1017/s0033291700050182.

24. Rossi A, Arduini L, De Cataldo S, Stratta P. Subjective response to neuroleptic medication: a validation study of the Italian version of the Drug Attitude Inventory (DAI). Epidemiol Psichiatr Soc. 2001;10(2):107-14. https://doi.org/10.1017/s1121189x00005182.

25. Kemp R, Hayward P, Applewhaite G, Everitt B, David A. Compliance therapy in psychotic patients: randomised controlled trial. BMJ. 1996;312(7027):345-9. https://doi.org/10.1136/bmj.312. 7027.345.

26. WMA. World Medical Association Declaration of Helsinki: ethical principles for medical research involving human subjects. JAMA. 2013;310(20):2191-4. doi:https://doi.org/10.1001/jama. 2013.281053.

27. StataCorp. Stata Statistical Software. Release 15.1 ed. College Station, TX: StataCorp LP; 2017.

28. Decuypere F, Sermon J, Geerts P, Denee TR, De Vos C, Malfait $\mathrm{B}$, et al. Treatment continuation of four long-acting antipsychotic medications in the Netherlands and Belgium: a retrospective database study. PLoS ONE. 2017;12(6):e0179049. https://doi.org/10. 1371/journal.pone.0179049.

29. Deslandes PN, Ward EH, Norris K, Sewell RD. Effectiveness of paliperidone long-acting injection in clinical practice. Ther Adv Psychopharmacol. 2018;8(5):139-45. https://doi.org/10.1177/ 2045125317753332.

30. Whale R, Pereira M, Cuthbert S, Fialho R. Effectiveness and predictors of continuation of paliperidone palmitate long-acting injection treatment: a 12-month naturalistic cohort study. J Clin
Psychopharmacol. 2015;35(5):591-5. https://doi.org/10.1097/jcp. 0000000000000385.

31. Attard A, Olofinjana O, Cornelius V, Curtis V, Taylor D. Paliperidone palmitate long-acting injection-prospective year-long follow-up of use in clinical practice. Acta Psychiatr Scand. 2014;130(1):46-51. https://doi.org/10.1111/acps.12201.

32. Young CL, Taylor DM. Health resource utilization associated with switching to risperidone long-acting injection. Acta Psychiatr Scand. 2006;114(1):14-20. https://doi.org/10.1111/j.1600-0447. 2006.00766.x.

33. Deslandes PN, Lewis A, Thomas A, Sewell RD. Risperidone long acting injection: findings of a 2-year retrospective follow-up study. Int J Psychiatry Clin Pract. 2009;13(4):298-302. https://doi.org/ 10.3109/13651500903046286.

34. Lammers L, Zehm B, Williams R. Risperidone long-acting injection in Schizophrenia Spectrum Illnesses compared to first generation depot antipsychotics in an outpatient setting in Canada. BMC Psychiatry. 2013;13:155. https://doi.org/10.1186/ 1471-244x-13-155.

35. Pilon D, Alcusky M, Xiao Y, Thompson-Leduc P, Lafeuille MH, Lefebvre $\mathrm{P}$, et al. Adherence, persistence, and inpatient utilization among adult schizophrenia patients using once-monthly versus twice-monthly long-acting atypical antipsychotics. J Med Econ. 2018;21(2):135-43. https://doi.org/10.1080/13696998.2017. 1379413.

36. Rittmannsberger H, Rosenleitner J, Malsiner-Walli G, Werl R, Rittmannsberger B, Yazdi K. Treatment duration with long-acting injectable antipsychotics after in-hospital initiation: a retrospective cohort study. J Clin Psychopharmacol. 2017;37(2):250-4. https://doi.org/10.1097/jcp.0000000000000672.

37. Taylor DM, Fischetti C, Sparshatt A, Thomas A, Bishara D, Cornelius V. Risperidone long-acting injection: a prospective 3 -year analysis of its use in clinical practice. J Clin Psychiatry. 2009;70(2):196-200.

38. Taylor DM, Sparshatt A, O'Hagan M, Dzahini O. Paliperidone palmitate: factors predicting continuation with treatment at 2 years. Eur Neuropsychopharmacol. 2016;26(12):2011-7. https:// doi.org/10.1016/j.euroneuro.2016.09.638.

39. Taylor DM, Young C, Patel MX. Prospective 6-month follow-up of patients prescribed risperidone long-acting injection: factors predicting favourable outcome. Int J Neuropsychopharmacol. 2006;9(6):685-94. https://doi.org/10.1017/s1461145705006309.

\section{Authors and Affiliations}

\section{Federico Bertolini ${ }^{1}$ (1) Giovanni Ostuzzi ${ }^{1} \cdot$ Michela Pievani $^{1} \cdot$ Andrea Agugliaa $^{2,3} \cdot$ Francesco Bartoli $^{4}$. Paola Bortolaso ${ }^{5}$. Camilla Callegari ${ }^{5}$. Mariarita Caroleo ${ }^{6}$. Giuseppe Carrà ${ }^{4,7} \cdot$ Mariangela Corbo $^{8}$. Armando D'Agostino $^{9,10}$. Pasquale De Fazio ${ }^{11}$. Fabio Magliocco ${ }^{6}$. Giovanni Martinotti ${ }^{8}$. Edoardo Giuseppe Ostinelli ${ }^{9}, 10$ - Marco Piero Piccinelli ${ }^{5}$. Federico Tedeschi ${ }^{1}$. Corrado Barbui ${ }^{1}$ - The STAR Network Investigators}

$1 \quad$ WHO Collaborating Centre for Research and Training in Mental Health and Service Evaluation; Department of Neuroscience, Biomedicine and Movement Sciences, Section of Psychiatry, University of Verona, Verona, Italy

2 Department of Neuroscience, Rehabilitation, Ophthalmology, Genetics, Maternal and Child Health, Section of Psychiatry, University of Genoa, Genoa, Italy

3 IRCCS Ospedale Policlinico San Martino, Genoa, Italy
4 Department of Medicine and Surgery, University of Milano Bicocca, Monza, Italy

5 Department of Medicine and Surgery, Division of Psychiatry, University of Insubria-ASST Sette Laghi, Varese, Italy

6 Department of Health Sciences, Psychiatric Unit, University Magna Græcia of Catanzaro, Catanzaro, Italy

7 Division of Psychiatry, University College of London, London, UK 
8 Department of Neuroscience, Imaging and Clinical Sciences, University "G. d'Annunzio", Chieti, Italy

9 Department of Health Sciences, Università degli Studi di Milano, Milan, Italy
10 Department of Mental Health, San Paolo Hospital, Milan, Italy

11 Azienda Ospedaliera Universitaria Mater Domini, University Magna Græcia of Catanzaro, Catanzaro, Italy 